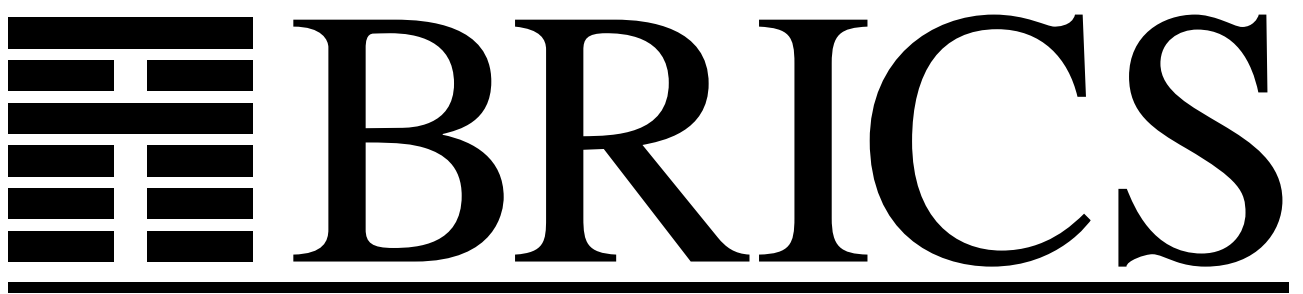

Basic Research in Computer Science

\title{
Type Checking with XML Schema in XACT
}

Christian Kirkegaard

Anders Møller

RS-05-31 
Copyright (c) 2005, Christian Kirkegaard \& Anders Møller. BRICS, Department of Computer Science University of Aarhus. All rights reserved.

Reproduction of all or part of this work is permitted for educational or research use on condition that this copyright notice is included in any copy.

See back inner page for a list of recent BRICS Report Series publications. Copies may be obtained by contacting:

\author{
BRICS \\ Department of Computer Science \\ University of Aarhus \\ Ny Munkegade, building 540 \\ DK-8000 Aarhus C \\ Denmark \\ Telephone: +4589423360 \\ Telefax: $\quad+4589423255$ \\ Internet: BRICS@brics.dk
}

BRICS publications are in general accessible through the World Wide Web and anonymous FTP through these URLs:

http://www.brics.dk

ftp: //ftp.brics.dk

This document in subdirectory RS / $05 / 31 /$ 


\title{
Type Checking with XML Schema in XACT
}

\author{
Christian Kirkegaard and Anders Møller* \\ BRICS $\dagger$ Department of Computer Science \\ University of Aarhus, Denmark \\ $\{\mathrm{ck}$, amoeller\}@brics.dk
}

September 2005

\begin{abstract}
We show how to extend the program analysis technique used in the $\mathrm{XACT}$ system to support XML Schema as type formalism. Moreover, we introduce optional type annotations to improve modularity of the type checking. The resulting system supports a flexible style of programming XML transformations and provides static guarantees of validity of the generated XML data.
\end{abstract}

\section{Introduction}

The overall goal of the XACT project is to integrate XML into general-purpose programming languages, in particular Java, such that programming of XML transformations can become easier and safer than with the existing approaches. Specifically, we aim for a system that supports a high-level and flexible programming style, permits an efficient runtime model, and has the ability to statically guarantee validity of generated XML data.

In previous papers, see $[14,13]$, we have presented the first steps of our proposal for a system that fulfills these requirements. Our language, ХАCT, is an extension of Java where XML fragments can be manipulated through a notion of XML templates using XPath for navigation. Static guarantees of validity are provided by a special data-flow analysis that builds on a lattice structure of summary graphs.

The existing XACT system has two significant weaknesses: first, it only supports DTD as schema language, and it is generally agreed that this language has insufficient expressiveness for modern XML applications; second, the dataflow analysis is a whole-program analysis that has poor modularity properties

\footnotetext{
*Supported by the Carlsberg Foundation contract number 04-0080.

${ }^{\dagger}$ Basic Research in Computer Science (www.brics.dk), funded by the Danish National Research Foundation.
} 
and hence does not scale well to larger programs. In this paper, we present an approach for attacking these issues.

\section{Contributions}

We have previously shown a connection between summary graphs and regular expression types [4, 10]. Also, it is known how regular expression types are related to RELAX NG schemas [7] and how schemas written in XML Schema [19, 2] can be translated into equivalent RELAX NG schemas [12]. We exploit these connections in this paper. Our main contributions are the following:

- We present a translation from XML Schema to summary graphs and an algorithm for validating summary graphs relative to schemas written in XML Schema, all via RELAX NG. This provides the foundation for using XML Schema as type formalism in XACT.

- We introduce optional typing in XACT so that XML template variables can be optionally typed with schema constructs (element names and simple or complex types). We show how this can lead to a validity analysis which is more modular, in the sense that it avoids iterating over the whole program.

Together, these improvements effectively remedy the weaknesses mentioned earlier. Furthermore, the results can be seen as an indication of the strength of summary graphs and the use of data-flow analysis for validating XML transformations.

As an additional contribution, we identify a subset of RELAX NG that is sufficient for translation from XML Schema and where language inclusion checking is tractable.

\section{Example}

The resulting XACT language can be illustrated by a small toy program that uses the new features. This program converts a list of business cards represented in a special XML language into XHTML, considering only the cards where a phone number is present:

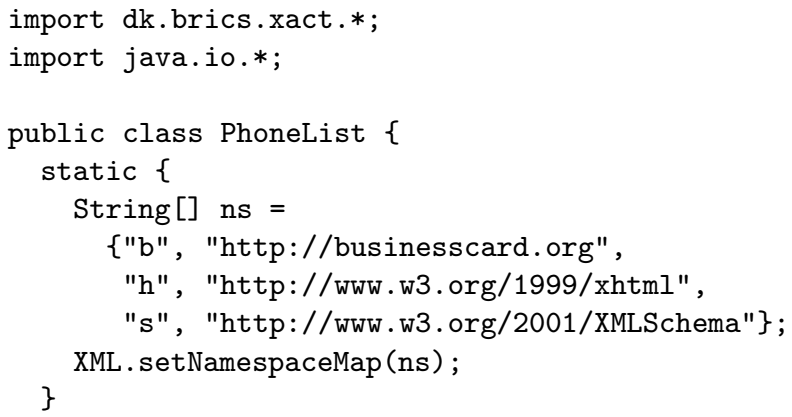




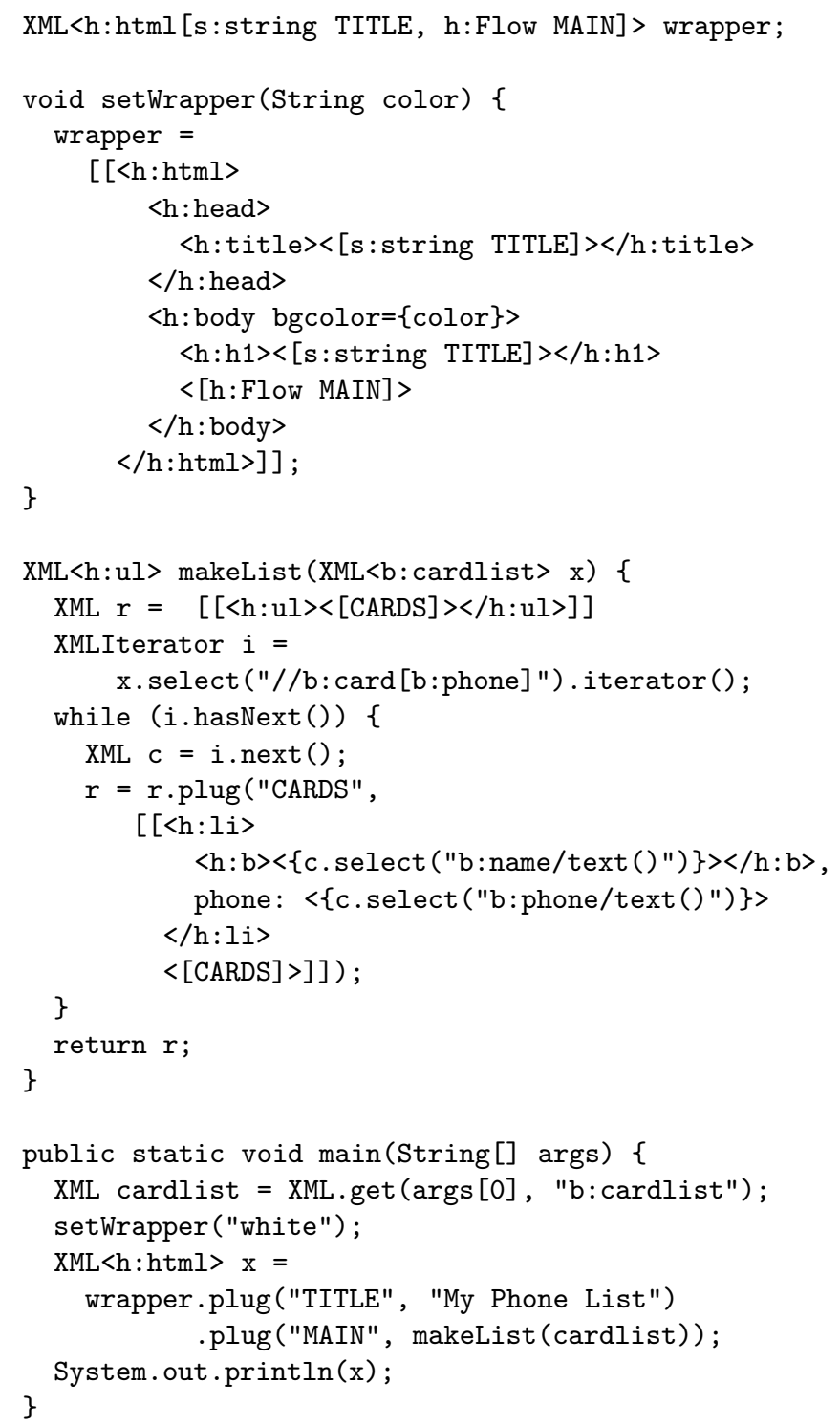

The general syntax for XML template constants and the meaning of the methods select, plug, get, and various others are described further in Section 2.

In the first part of the program, some global namespace declarations are made. Schemas for these namespaces are supplied externally (the schema for the business card XML language is shown in Section 3). Then a field wrapper is defined, holding an XML template that must be an html tree, potentially with TITLE gaps and MAIN gaps, which may occur in place of fragments of type string and Flow, respectively (all of appropriate namespaces). The method 
setWrapper assigns such an XML template to the wrapper field. This template has two gaps named TITLE and one named MAIN. Additionally, it has one code gap where the value of the color parameter is inserted. The method makeList iterates through a list of card elements that have phone children and builds an XHTML list. The method main loads in an XML document containing a list of business card, invokes the setWrapper method, then constructs a complete XHTML document by plugging values into the TITLE and MAIN gaps using the makeList method, and finally outputs this document.

As an example, the program transforms the input

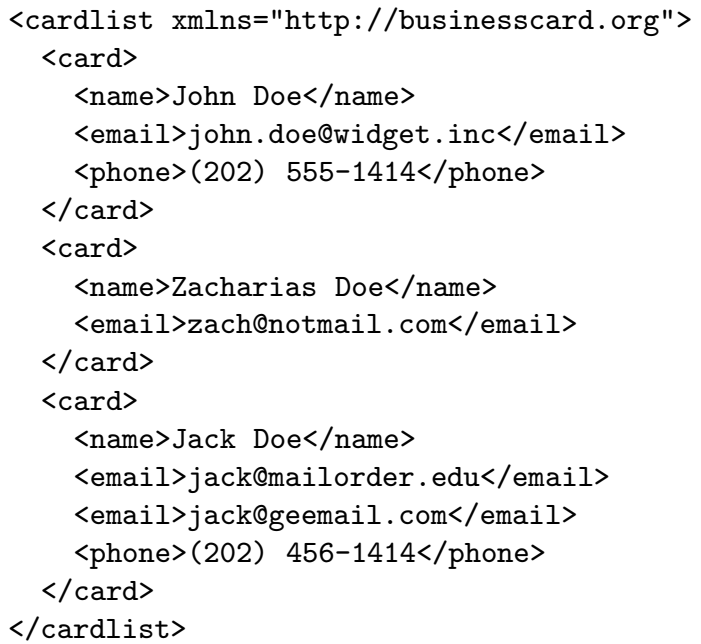

into an XHTML document that looks as follows:

\section{My Phone List \\ - John Doe, phone: (202) 555-1414 \\ - Jack Doe, phone: (202) 456-1414}

Note that some XML variables in the program are declared by the type XML, which represents all possible XML templates, and others use a more constrained type, such as, the declaration of wrapper or the signature of makeList. XACT now allows the programmer to combine these two approaches. The static type checker uses data-flow analysis to reason about variables that are declared using the former approach, and it conservatively checks that the annotated types are preserved by execution of the program. For this program, one consequence is that the makeList method, whose signature is fully annotated, can be type checked separately, and invocations of this method can be type checked without considering its body. (We discuss fields and side-effects in Section 7.) Also note that the type checker can now reason about XML Schema types rather that being limited to DTD. 


\section{Related Work}

There are numerous other projects having similar goals as XACT; the paper [17] contains a general survey of different approaches. The ones that are most closely related to ours are XJ [9], C $\omega[1]$, and XDuce and its descendants [10]. XACT is notably different in two ways: first, although variants of XML templates are widely used in Web application development frameworks, this paradigm is not supported by other type-safe XML transformation languages, which typically allow only bottom-up XML tree construction; second, the annotation overhead is minimal since schema types are only required at input and output, whereas the others require schema type annotations at all XML variable declarations. We believe that both aspects in many cases makes the XACT programming style more flexible. Furthermore, our data-flow analysis also tracks string operations [6], which enables it to reason about validity of attribute values and character data - in contrast, the type checkers in the other systems generally only focus on the structural parts of XML languages. (In fact, an additional consequence of the extensions described here is that our static analyzer can also model computed names of elements and attributes, however, we leave this idea to future work.)

With the extensions proposed in this paper, XACT becomes closer to XJ [9], which also uses XML Schema as type formalism and XPath for navigation. Still, our use of optional type annotations avoids a problem that can make the XJ type checker too rigid: with mandatory type annotations at all variable declarations in XJ, it is impossible to type check a sequence of operations that temporarily invalidate data. The types that are involved in XML transformations are often exceedingly complicated and difficult to write down, and types for intermediate results often do not correspond to named constructs in preexisting schemas. The benefits of type annotations are that they can serve as documentation in the programs and they can improve performance of type checking. By now supporting optional annotations, XACT gets the best from the two worlds.

Moreover, XJ represents XML data as mutable trees, which incurs a need for expensive runtime checks to preserve data validity. In XJ, subtyping is nominal, whereas our approach gives semantic (or structural) subtyping. A discussion of subtyping can be found in [8]. Note that although XML Schema does contain mechanisms for declaring subtyping relationships nominally, the choice of supporting XML Schema as type formalism in XACT does not force us to use nominal subtyping. We use schemas only as notation for defining sets of $\mathrm{XML}$ values - the internal structure of the notation being used is irrelevant.

The XDuce language family is based on the notion of regular expression types. As mentioned earlier, a connection between regular expression types and a variant of the summary graphs used in our program analysis is shown in [4]. Also, the formal expressiveness of regular expression types and RELAX NG both correspond to that of regular tree languages. We return to these relations in Sections 5 and 6. As XACT, the XтATIC language [8], which is one of the descendants of XDuce, incorporates XML into an object-oriented language in an immutable style. 
The $\mathrm{C} \omega$ language adds XML support to $\mathrm{C}^{\sharp}$ by combining structural sequences, unions, and products with objects and simple values. The basic features of XML Schema may be encoded in the type system, however little documentation of this is available. Rather than use full XPath for navigation in $\mathrm{XML}$ trees as in XACT, $\mathrm{C} \omega$ uses a reminiscent notion of generalized member access that is closer to ordinary programming notation.

The type annotations we introduce are reminiscent of the notion of programmer-designer contracts proposed in [3]. In both cases, static declarations constrain how XML templates may be combined in the programs.

The paper [18] contains a useful classification of schema languages in terms of categories of tree grammars: DTD corresponds to local tree grammars where the content model of an element can only depend on the name of the element; XML Schema corresponds to the larger category of single-type tree grammars where elements that are siblings and have the same name must have identical content models; and RELAX NG corresponds to the even more general category of regular tree grammars, which is equivalent to tree automata. With our new results, XACT supports single-type tree grammars as type formalism.

\section{Overview}

In Sections 2 and 3 we begin by briefly recapitulating the design of XACT and RELAX NG, and we characterize a subset of RELAX NG, called Restricted RE$L A X N G$, that we will use as an intermediate language in the program analysis. Then, in Section 4 we introduce a variant of summary graphs. In Sections 5 and 6 we explain how schemas written in XML Schema can be converted into summary graphs via Restricted RELAX NG, how to check validity of summary graphs relative to Restricted RELAX NG schemas, and how these results can be used in XACT to provide static guarantees of XML transformations. In Section 7 we introduce optional typing using XML Schema constructs and discuss the resulting language design. Finally, we present our conclusions in Section 8.

Note that we here report on work in progress, and not all of what we present has yet been implemented and tested in practice so we cannot at this stage present experimental results.

\section{The $\mathrm{X}_{\mathrm{ACT}}$ Programming Language}

We begin with a brief overview of the XАCT language as it looks before adding our new extensions. In XАCT, XML data is represented as templates, which are well-formed XML fragments that may contain gaps in place of elements or attribute values. A gap is either a name or a piece of code that evaluates to a string or an XML template. As an example, the following XML template contains four gaps: two named TITLE, one named MAIN, and one containing the expression color:

<h: html>

$<\mathrm{h}$ : head $>$ 


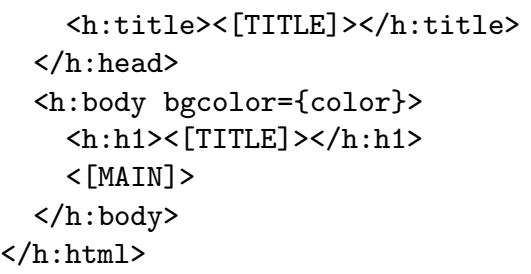

The special immutable class XML corresponds to the set of all possible XML templates. The central operations on this class are the following:

constant: a static method that creates a template from a constant string (the syntax [ffo]] is sugar for XML.constant ("foo"));

plug: inserts a given string or template into all gaps of a given name in this template;

select: returns the sub-templates of this template that are selected by a given XPath expression;

get: a static method that creates a template from a non-constant string and checks (at runtime) that it is valid relative to a given constant schema type;

cast: performs a runtime check of validity of this template relative to a given constant schema type;

analyze: instructs the static type checker to verify that this template will always be valid relative to a given schema type when the program runs (remaining gaps are here treated as empty subtrees); and

toString: converts this template to its textual representation.

A schema type is the name of an element (or, with our extension from DTD to XML Schema, a simple type or a complex type) that is declared in a schema. The language of a schema type is defined as the set of XML documents (or document fragments) that are valid relative to the schema type. Note that in this version of XACT, before incorporating the extensions suggested in this paper, schema types appear only at get, cast, and analyze operations. In particular, declarations use the general type XML.

The primary job of the static type checker is to verify that only valid XML data can occur at program locations marked by analyze operations, under the assumption that get and cast operations always succeed. (It also checks properties of plug and select operations, which is less relevant here.)

\section{Defining a Subset of RELAX NG}

A RELAX NG schema [7] is essentially a top-down tree automaton that accepts a set of valid XML trees. It is described by a grammar consisting of recursively defined patterns of various kinds, including the following: element matches one 
element with a given name and with contents and attributes described by a subpattern; attribute similarly matches an attribute; text matches any character data or attribute value; group, optional, zeroOrMore, oneOrMore, and choice correspond to concatenation, zero or one occurrence, zero or more occurrences, one or more occurrences, and union, respectively; empty matches the empty sequence of nodes; and notAllowed corresponds to the empty language. In addition, the pattern interleave matches all possible mergings of the sequences that match its sub-patterns.

Note that attributes are described in the same expressions as the content models. Still, attributes are considered unordered, as always in XML, and syntactic restrictions prevent an attribute name from occurring more than once in any element. Mixing attributes and contents in this way is useful for describing attribute-element constraints.

To ensure regularity, there is an important restriction on recursive pattern definitions: recursion is only allowed if passing through an element pattern.

Element and attribute names can be described with name classes, which can consist of lists of possible names and wildcards that match all names, potentially restricted to a certain namespace or excluding certain specific names.

To describe datatypes more precisely than with the text pattern, RELAX NG relies on an external language, usually the datatype part of XML Schema. Using the data pattern, such datatypes can be referred to, and datatype facets can be constrained by a parameter mechanism.

Furthermore, RELAX NG contains various modularization mechanisms, which we can ignore here. As all other type-safe XML transformation languages, we also ignore ID and IDREF attributes from DTD and the equivalent compatibility features in RELAX NG.

As mentioned in the introduction, we handle XML Schema via a translation to RELAX NG [12], thus using RELAX NG as a convenient intermediate language that avoids the many complicated technical details of XML Schema. However, we only use a subset of RELAX NG, which we call Restricted RELAX $N G$, being characterized as follows:

- for every element pattern $p$, any two element patterns that correspond to possible children of $p$ and have non-disjoint name classes must have the same content;

- attribute patterns can only occur directly inside element patterns or in optional, zeroOrMore, or oneOrMore patterns inside element patterns, however, zeroOrMore and oneOrMore are only allowed if the attribute pattern has an infinite name class; and

- interleave patterns can only occur directly inside element patterns and can only contain element and text patterns, potentially enclosed by optional patterns, and the only allowed siblings of interleave patterns are attribute patterns and optional, zeroOrMore, and oneOrMore patterns that contain attribute patterns.

(We here consider ref patterns as abbreviations of the patterns being referred 
to.) The first property limits the notation to single-type tree grammars, the second prevents context sensivity of attribute patterns, and the third makes it easier to check inclusion of interleave patterns.

Restricted RELAX NG has two important properties: first, it is sufficient for the translation from XML Schema; second, it makes the summary graph validation in Section 6 significantly more tractable.

By the translation to Restricted RELAX NG, a schema type corresponds to a pattern definition:

- an element declaration corresponds to an element pattern;

- a simple type corresponds to a pattern, which we call a simple-type pattern, that can only contain the constructs data and choice (and also list and value, which we otherwise ignore here for simplicity); and

- a complex type corresponds to a pattern, which we call a complex-type pattern, that consists of two sub-patterns - one describing a content model and one describing attributes.

We use this observation in Section 6.

The following schema written in XML Schema may be used to describe the input to the example program shown in Section 1:

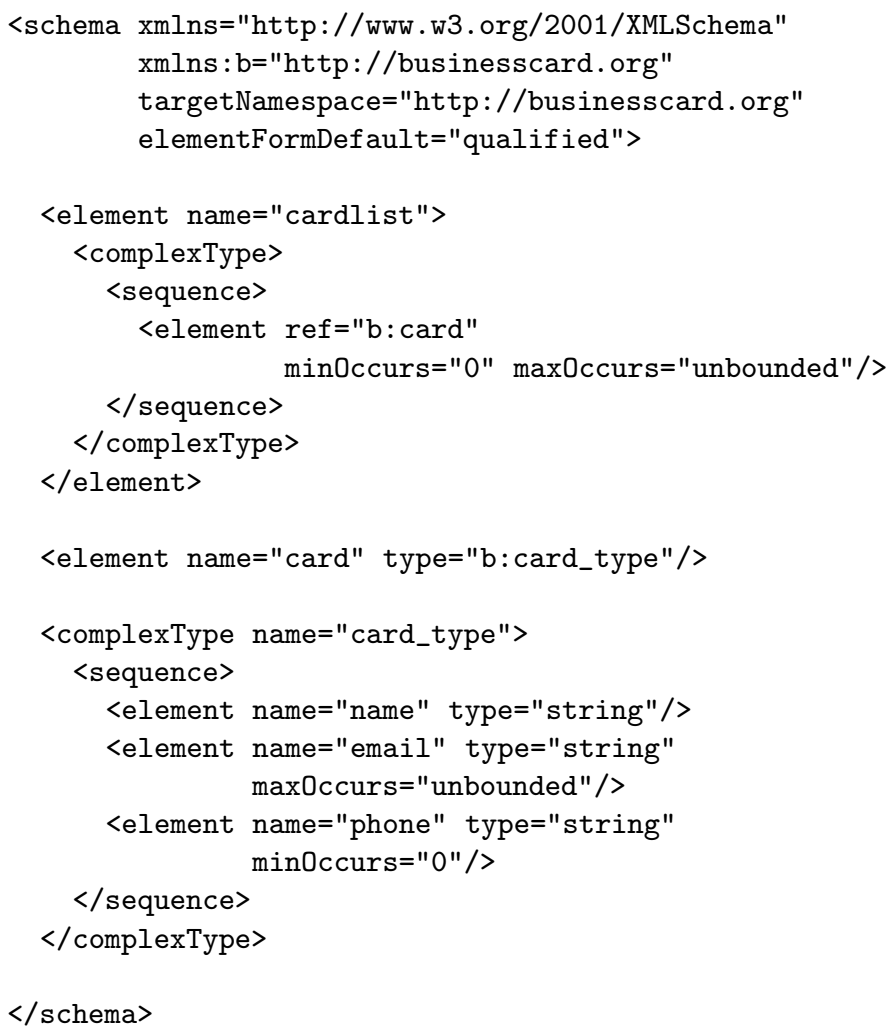

$</$ schema $>$ 
Assuming cardlist as root element name, this can be translated into the following Restricted RELAX NG schema (here using the compact RELAX NG syntax):

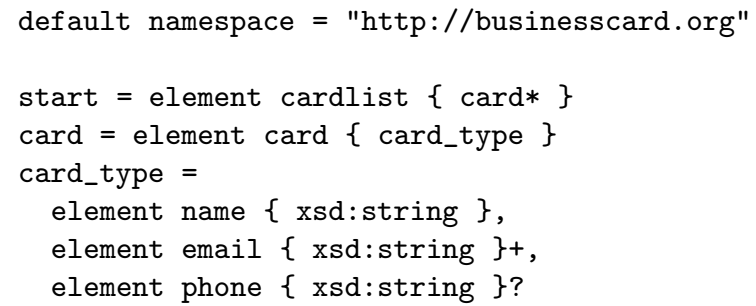

The translation from XML Schema to Restricted RELAX NG is exact and the size of the output schema is proportional to the size of the input schema.

\section{Summary Graphs in Validity Analysis}

The static type checker in XАCT works in two steps. First, a data-flow analysis of the whole program is performed, using the standard data-flow analysis framework [11] but with a highly specialized lattice structure where abstract values are summary graphs. A summary graph is a finite representation of a potentially infinite set of XML templates, much like a schema but tailor-made for use in the program analysis [14]. Second, when the fixed point has been computed, we check that the sets of templates represented by the resulting summary graphs are valid relative to the respective schemas.

To allow a smooth integration of XML Schema as a replacement for DTD, we slightly modify the definition of summary graphs as explained below and change the summary graph validation algorithm accordingly and to work with Restricted RELAX NG (the old algorithm supported DTD via an embedding into DSD2 [16]).

A summary graph, as it is defined in [14], has two parts: one that is set up for the given program and remains fixed during the iterative data-flow analysis, and one that changes monotonically during the analysis.

The fixed part contains finite sets of nodes of various kinds: element nodes $\left(N_{\mathcal{E}}\right)$, attribute nodes $\left(N_{\mathcal{A}}\right)$, chardata nodes $\left(N_{\mathcal{C}}\right)$, and template nodes $\left(N_{\mathcal{T}}\right)$. These node sets are determined by the use of schemas, template constants, and XML operations. The former three sets represent the possible elements, attributes, and chardata sequences that may arise when running the program. The template nodes represent sequences of template gaps, which either occur explicitly in template constants or implicitly due to XML operations or schemas. Additionally, the fixed part specifies a number of maps: name assigns a name to each element node and attribute node; attr $: N_{\mathcal{E}} \rightarrow 2^{N_{\mathcal{A}}}$ associates attribute nodes with element nodes; contents $: N_{\mathcal{E}} \rightarrow N_{\mathcal{T}}$ connects element nodes with descriptions of their contents; and gaps $: N_{\mathcal{T}} \rightarrow G^{*}$ associates a sequence of gap names from a finite set $G$ with each template node.

The changing part of a summary graph consist of: 
- a set of root nodes $R \subseteq N_{\mathcal{E}} \cup N_{\mathcal{T}}$;

- template edges $T \subseteq N_{\mathcal{T}} \times G \times\left(N_{\mathcal{T}} \cup N_{\mathcal{E}} \cup N_{\mathcal{C}}\right)$;

- string edges $S: N_{\mathcal{C}} \cup N_{\mathcal{A}} \rightarrow R E G$ where $R E G$ are all regular string languages over the Unicode alphabet; and

- a gap presence map $P: G \rightarrow 2^{N_{\mathcal{A}} \cup N_{\mathcal{T}}} \times 2^{N_{\mathcal{A}} \cup N_{\mathcal{T}}} \times \Gamma \times \Gamma$ where $\Gamma=$ $2^{\{\text {OPEN, CLOSED }}$.

The language of a summary graph is intuitively the set of XML templates that can be obtained by unfolding it, starting from a root node and plugging elements, templates, and strings into gaps according to the edges. A template edge $\left(n_{1}, g, n_{2}\right) \in T$ informally means that $n_{2}$ may be plugged into the $g$ gaps in $n_{1}$, and a string edge $S(n)=L$ means that every string in $L$ may be plugged into the gap in $n$. The gap presence map, which we will not explain in further detail here, is needed during the data-flow analysis to determine where template gaps and attribute gaps occur. (For the curious reader, this is all formalized in [14].) We also define the language of an individual node $n$ in a summary graph: this is simply the language of the modified summary graph where $R$ is set to $\{n\}$.

We now introduce two small modifications:

1. We let the name function return a regular set of names, rather than a single name. This will be used to more easily model name classes in Restricted RELAX NG. The definition of unfolding is generalized accordingly: unfolding an element node $n$ yields an element whose name can be any string in name $(n)$, and similarly for attribute nodes. In case an unfolding leads to an element with two attributes of the same name, one of them is chosen arbitrarily and overrides the other.

To accommodate attribute declarations that have infinite name classes and are repeated using zeroOrMore or oneOrMore, we define the unfolding of an attribute node $n$ where name $(n)$ is infinite such that it may produce more than one attribute.

2. We distinguish between two kinds of template nodes: sequence nodes and interleave nodes. The former have the meaning of the old template nodes; the latter will be used to model interleave patterns. We define the unfolding of an interleave node as all possible interleavings of the unfoldings of its gaps.

Reflecting the limitation on the interleave construct in Restricted RELAX NG, we require that template edges from interleave nodes can only end in element nodes or chardata nodes (not in other template nodes). Also, template edges cannot end in interleave nodes (thus, interleave nodes can only occur as roots and as immediate content of element nodes).

As an example (borrowed from [14]), we can define a summary graph whose language is the set of ul lists with zero or more $l i$ items that each contain a 
string from some language $L$. Assume that the fixed structure is given by $N_{\mathcal{E}}=$ $\{1,4\}, N_{\mathcal{A}}=\emptyset, N_{\mathcal{T}}=\{2,3,5\}$ (where all three are sequence nodes), $N_{\mathcal{C}}=\{6\}$, contents $(1)=2$, contents $(4)=5, \operatorname{attr}(1)=\operatorname{attr}(4)=\emptyset$, name $(1)=\{\mathrm{ul}\}$, name $(4)=\{1 i\}, \operatorname{gaps}(2)=i$ tems, gaps $(3)=g \cdot i$ tems, and gaps $(5)=$ text. The remaining components are as follows:

$$
\begin{aligned}
& R=\{1\} \\
& T=\{(2, \text { items }, 3),(3, \text { items }, 3),(3, g, 4),(5, \text { text }, 6)\} \\
& S(6)=L
\end{aligned}
$$

(For simplicity, we ignore the gap presence map.) This can be illustrated as follows:

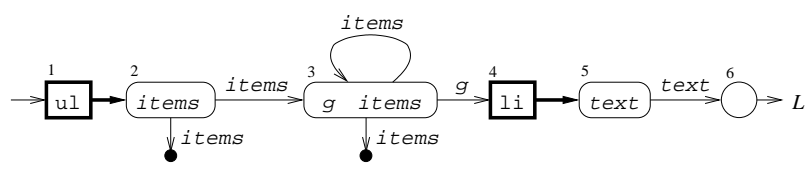

The boxes represent element nodes, rounded boxes are template nodes, the circle is a chardata node, and the dots represent potentially open template gaps.

For a given program, the family of summary graphs forms a finite-height lattice, which is used in the data-flow analysis. To determine the regular string languages used in the string edges, we use a separate program analysis that provides conservative approximations of the possible values of all string expression in the given program [6]. The data-flow transfer functions for operations remain as explained in [14] with only negligible changes as consequence of the modifications of the summary graph definition, the only exceptions being the ones we address in the following section.

With the generalization of the name function, we can in fact now easily model computed names of elements and attributes - provided that we add operations for this in the XML class, of course, and we leave this to future work.

\section{A Translation from Restricted RELAX NG to Summary Graphs}

To define the transfer functions for the operations get and cast, we need an algorithm for translating the given schema type into a summary graph that has the same language. In [14], it is shown how this can be done for DTD schemas; we now present a modified algorithm that supports Restricted RELAX NG and then rely on the translation from XML Schema to Restricted RELAX NG to map from schema types to patterns.

Intuitively, this translation is straightforward: we may simply view summary graphs as a graphical representation of Restricted RELAX NG patterns, provided that we ignore the gap presence component of the summary graphs and the regularity requirement in Restricted RELAX NG. Due to the connection between RELAX NG and regular expression types, this translation can also 
be seen as a variant of the translation between regular expression types and summary graphs shown in [4].

Given a Restricted RELAX NG pattern, we construct a summary graph fragment as follows:

- First, we observe that each name class, each data pattern, and the text pattern all define regular string languages. (Namespaces are handled by expanding qualified names according to the applicable namespace declarations.)

- For an element pattern, we exploit the syntactic restrictions described in Section 4. An element pattern always consists of three parts: a name class, a content model, and a collection of attribute declarations. Thus, we convert it to an element node $e$ and a template node $t$ with contents $(e)=$ $t$. We define name $(e)$ as the regular string language corresponding to the name class. The attribute declarations are converted recursively into attribute nodes (as explained below), and $\operatorname{attr}(e)$ is set accordingly. The content model is converted recursively into a summary graph fragment rooted by $t$.

- An attribute pattern is converted into an attribute node $a$. We define name $(a)$ in the same way as for element patterns, and $S(a)$ is set to the regular string language corresponding to the sub-pattern describing the attribute values. If the attribute is declared as optional using the optional pattern, the gap presence map is set to record this (as in [14]).

- For patterns describing content models of elements, the patterns text, group, optional, zeroOrMore, oneOrMore, choice, and empty are handled exactly as the equivalent constructs in DTD content model definitions in the way explained in [14]. Intuitively, each pattern corresponds to a tiny summary graph fragment that unfolds to the same language. A data pattern becomes a chardata node $s$ where $S(s)$ is the corresponding regular string language. The interleave pattern is translated in the same way as group, except that an interleave node is used instead of a sequence node.

- Finally, the notAllowed pattern can be modeled as a template node $t$ where $\operatorname{gaps}(t)=g$ for some gap name $g$ and $t$ has no outgoing template edges.

The set of root nodes $R$ contains the single node that corresponds to the whole pattern being translated. Recursion in pattern definitions simply results in loops in the summary graph. The constructs from RELAX NG that we have omitted in the description in Section 3 can be handled in a similar way as those mentioned here. Note that the translation is exact: the language of the pattern is the same as the language of the resulting summary graph.

As an example, translating the pattern

element ul \{ element li \{xsd:integer \}* \} 
results in the summary graph shown in Section 4, assuming that $L$ is the language of strings that match xsd:integer.

\section{$6 \quad$ Validating Summary Graphs}

When the data-flow analysis has computed a summary graph for each XML expression in the XACT program, we check for each analyze operation that the language of its summary graph is included in the language of the specified schema type. If the check fails, appropriate validity warnings are emitted. The entire analysis is sound: if no validity warnings show up, the programmer can be sure that, at runtime, the XML values that appear at the program points marked by analyze operations will be valid relative to the given schema types.

The old summary graph analyzer used in XACT is described in [5]. That algorithm, which supports DTD through an embedding into DSD2, as mentioned earlier, has proven successful in practice. We here describe a variant that works with Restricted RELAX NG instead of DSD2.

Given a summary graph node $n \in N_{\mathcal{E}} \cup N_{\mathcal{T}}$ and a Restricted RELAX NG pattern $p$ where $p$ is an element pattern, a simple-type pattern, or a complex-type pattern (as defined in Section 3), we wish to determine whether the language of $n$ is included in the language of $p$.

We begin by considering the case where $n$ is not an interleave node and $p$ is not an interleave pattern. First, a context-free grammar $C$ is constructed from the part of the summary graph that is rooted by $n$ and where element and chardata nodes are considered as terminals (that is, nested element and chardata nodes are not considered here). Each of the chardata node terminals $c$ is then replaced by a regular grammar equivalent to $S(c)$. If $C$ is not linear, we apply a regular over-approximation [15] (which we also use in [6]). Thus, we have a regular string language $L_{n}$ over element nodes and Unicode characters that describes the possible unfoldings of $n$. Similarly, $p$ defines a regular string language $L_{p}$ over element patterns and Unicode characters. To obtain a common vocabulary, we now replace each element node $n^{\prime}$ in $L_{n}$ by $\left\langle\right.$ name $\left.\left(n^{\prime}\right)\right\rangle$ (where $\langle$ and $\rangle$ are some otherwise unused characters), and similarly for the element patterns in $L_{p}$. Then, we check that $L_{n}$ is included in $L_{p}$ with standard techniques for regular string languages. If this check fails, a suitable validity error message is generated. Otherwise, for each pair $\left(n^{\prime}, p^{\prime}\right)$ of an element node in $L_{n}$ and an element pattern in $L_{p}$ where name $\left(n^{\prime}\right)$ and name $\left(p^{\prime}\right)$ are nondisjoint, we perform two checks: first, we check recursively that the language of contents $\left(n^{\prime}\right)$ is included in the language of the content model of $p^{\prime}$; second, we check that the attributes of $n^{\prime}$ match those on $p^{\prime}$. The latter check is made as follows: For each attribute node $a \in \operatorname{attr}\left(n^{\prime}\right)$, each name $x \in$ name $(a)$, and each value $y \in S(a)$, a corresponding attribute pattern must occur in $p^{\prime}$ - that is, one where $x$ is in the language of its name class and $y$ is in the language of its sub-pattern; also, if $a$ is marked as optional by the gap presence map, the attribute pattern must be enclosed by an optional pattern, and conversely, attribute patterns occurring in $p^{\prime}$ that are not enclosed by optional patterns 
must correspond to one of the non-optional attribute nodes. Again, a suitable validity error message is generated if the check fails.

If $n$ is an interleave node and $p$ is an interleave pattern, we exploit the restrictions on these constructs and check for each gap $g_{i}$ in gaps $(n)=g_{1} \ldots g_{k}$ that a sub-pattern $p^{\prime}$ of $p$ can be chosen such that there is a template edge $\left(n, g_{i}, n^{\prime}\right)$ where the language of $n^{\prime}$ is included in the language of $p^{\prime}$ and each of the sub-patterns of $p$ is chosen exactly once. Here, we can ignore chardata nodes and text patterns, except that we require that $p$ contains a text pattern if a chardata node occurs.

The remaining cases where $n$ is an interleave node but $p$ is not an interleave pattern or $n$ is not an interleave node but $p$ is an interleave pattern can be handled by combinations of the algorithms explained above.

To avoid redundant computations (and to ensure termination, in case of loops in the summary graph or recursive definitions in the schema) we apply memoization such that a given pair $(n, p)$ is only processed once. If a loop is detected, we can coinductively assume that the inclusion holds.

With this algorithm, we check for each root node $n \in R$ that its language is included in the language of the pattern corresponding to the given schema type.

As an example of the case with an element node and an element pattern, let $n$ be element node 1 in the summary graph from Section 4 and let $p$ be the pattern shown in Section 5:

$p=$ element ul $\{$ element li $\{\mathrm{xsd}$ :integer $\} *\}$

The context-free grammar for $L_{n}$ has the following productions (where only $N_{4}$ is a terminal):

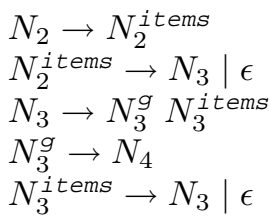

This grammar is linear, so the regular approximation is not applied. The pattern $p$ contains a single sub-pattern

$p^{\prime}=$ element $1 \mathrm{i}\{\mathrm{xsd}$ :integer $\}$

and by recursively comparing node 4 and $p^{\prime}$ we find out that the language of node 4 is included in the language of $p^{\prime}$. We now see that $L_{n} \subseteq L_{p}$, so we conclude that the language of element node 1 is in fact included in the language of the pattern.

With the exception of the regular approximation of the context-free grammars mentioned above, the inclusion check is exact. Also, since the schemas already define only regular languages, the approximation can only cause a loss of precision if the XML transformation defined by the XACT program introduces non-regularity in the summary graphs, and our experience from [14] and 
[5] indicate that this rarely results in false errors. In particular, the trivial identity function, which inputs XML data using get with some schema type and immediately after applies analyze with the same schema type, is guaranteed to type check without warnings for any schema type. Moreover, we could replace the approximation by an algorithm that checks inclusion of a context-free language in a regular language, if full precision is considered more important than performance.

An obvious alternative approach to the algorithm explained above would be to exploit the connection with regular expression types and apply the results from the XDuce project for checking subtyping between regular expression types [10]. Our main argument for choosing the algorithm explained above is that it has been shown earlier that this approach is efficient for XACT programs. Still, the relation between these different inclusion checking algorithms is worth a further investigation.

As an interesting side-effect of our approach, we get an inclusion checker for Restricted RELAX NG and hence also for XML Schema: given two schemas, $S_{1}$ and $S_{2}$, convert $S_{1}$ to a summary graph $S G$ using the algorithm described in Section 5 and then apply the algorithm presented above on $S G$ and $S_{2}$. (Alternatively, the algorithm presented above could be modified to work directly with Restricted RELAX NG schemas instead of summary graphs.)

\section{Optional Type Annotations}

We will now extend XACT with optional type annotations such that programmers may declare the intended schema types for XML template variables, method parameters, and return values. Besides being useful as in-lined documentation of programmer intentions, type annotations can lead to better modularity properties of the validity analysis.

Every XML type may now optionally be annotated in the following way where $S$ and $T_{1}, \ldots, T_{n}$ are schema types and $g_{1}, \ldots, g_{n}$ are gap names:

$$
\mathrm{XML}<S\left[T_{1} g_{1}, \ldots, T_{n} g_{n}\right]>
$$

The semantics of an annotated type is the language described by $S$ under the assumption that every occurrence of gap $g_{i}$ has been plugged with a value in the language of schema type $T_{i}$.

In XML template constants, every template gap must now have the form $<\left[\begin{array}{ll}T & g\end{array}\right]>$, where $T$ is a schema type and $g$ is the gap name. This allows us to, at runtime, tag each gap $g$ in an XML template with a schema type.

In gap annotations in XML declarations and template costants, we permit Kleene star of a schema type, $T *$, meaning that the gap can be filled with a sequence of values from the language of $T$. Kleene star annotations are occasionally needed because we cannot always find existing schema types for sequences of values. As an example, the XML Schema description of XHTML has no named content type describing a sequence of $1 i$ elements. Theoretically, we could permit type annotations to be arbitrary regular expressions over schema types or 
even small inlined XML Schema fragments, but we have not yet observed the need for this.

Every assignment of an XML template $v$ to a variable $x$ whose type annotation is $t=S\left[\begin{array}{llll}T_{1} & g_{1}, \ldots, T_{n} & g_{n}\end{array}\right]$ must, at runtime, satisfy three constraints:

- All gaps occurring in $v$ must be declared in $t$.

- For every gap $g$ occurring in $v$, the language of its type tag must be included in the language of the schema type for $g$ as declared in $t$.

- The value $v$ must, under the assumption that all gaps were plugged according to their type tags, belong to the language of $S$.

We put similar constraints on return statements and method invocations, except that for return statements the return value is compared with the declared return type, and for method invocations every actual parameter value is compared with the corresponding declared parameter type. Moreover, every plug operation must respect gap tags, that is, the value being plugged in to a gap $g$ must belong to the language of the tag of $g$.

The following describes a modification of our existing static program analysis to support checking of the extra constraints introduced by annotations.

First, the abstract representation of sets of XML templates is extended to also keep track of the declared schema types of gap names. For a given XACT program, we let $\mathcal{T}$ denote the finite set of all types mentioned by gap annotations in template constants, and we introduce a new summary graph component $D$ : $G \rightarrow \mathcal{T}$ mapping gap names to their declared type. The language of a summary graph is not affected by this change.

This leads to extending the data-flow transfer function for the constant operation to generate a summary graph with mappings $D(g)=T$ for every gap $<T$ $g>$ occurring in the given XML template constant. (A simple syntactical check ensures that in each template constant all gaps of the same name are declared with identical schema types.) The transfer function for the plug operation simply unions the $D$ mappings of its arguments. (Conflicts are avoided by a check mentioned below.) All other transfer functions act as the identity on the new $D$ component.

To ensure type consistency of variables declared with annotated XML types, we must validate all assignments to such variables. We check, using the validation algorithm described in Section 6, that the language of the inferred summary graph for the right-hand side of an assignment is a subset of the language permitted by the schema type annotation. However, this inclusion check is modified to treat gaps as if they were plugged with values corresponding to their declared types. More precisely, for every gap $g$ in the inferred summary graph we apply the algorithm described in Section 5 to construct a summary graph fragment $S G_{g}$ corresponding to the schema type $D(g)$ and then add template edges from all occurrences of $g$ to the roots of $S G_{g}$.

To ensure type consistency of template gaps, we perform an additional check of every $x \cdot \operatorname{plug}(g, y)$ operation using the summary graphs $S G_{x}$ and $S G_{y}$ inferred by the data-flow analysis for $x$ and $y$, respectively. First, we check that 
the language of $S G_{y}$ is a subset of the language of $D_{x}(g)$ declared for $g$ in $S G_{x}$ using the inclusion algorithm presented in Section 6 . Then, we check that all gap names $h$ occurring in both $S G_{x}$ and $S G_{y}$ are declared with identical types, that is, $D_{x}(h)=D_{y}(h)$.

As a product of the guaranteed type consistency of variables declared with annotated XML types, reading from a variable can now use the declared type instead of the inferred one. More precisely, for every read from an XML typed variable $x$ we normally use an inferred summary graph to describe the set of possible template values at that program point, but now, since all assignments to $x$ have already been checked for validity with respect to the declared schema type for $x$, we can instead apply the algorithm from Section 5 to obtain the summary graph corresponding to the declared schema type.

Note that the support for type annotations leads to a programming style where the explicit analyze operation is rarely needed - instead, one may request a static type check by assigning to an annotated variable. This is the style required in other XML transformation languages.

It is well-known that type annotations in programming languages enable more modular type checking. A component, whose interface is fully annotated, can be type checked independently of its context, and type checking the context can be performed without considering the body of the component. In our setting, this, for example, corresponds to methods where all XML typed parameters and return types are annotated, and further, every non-local assignment and read within the method body involves fields declared with annotated types (the latter to constrain side-effects through field variables). As discussed in Section 1, annotations also have drawbacks, however, in XACT, type annotations are optional. This allows the programmer to mix annotated and unannotated XML types to get the best from both worlds.

\section{Conclusion}

We have presented an approach for generalizing the XACT system to support XML Schema as type formalism and permit optional type annotations. Compared with other programming languages for type-safe XML transformations, type annotations are permitted but not mandatory, which allows the programmer to balance between the pros and cons of type annotations.

The extension to XML Schema takes advantage of connections between XML Schema, RELAX NG, and summary graphs. In particular, it involves a tractable subset of RELAX NG that we use as an intermediate language in the static analysis.

We are working on making the ideas presented in this paper available in the next version of the XАCT implementation. 


\section{References}

[1] Gavin Bierman, Erik Meijer, and Wolfram Schulte. The essence of data access in $\mathrm{C} \omega$. In Proc. 19th European Conference on Object-Oriented Programming, ECOOP '05, volume 3586 of LNCS. Springer-Verlag, July 2005.

[2] Paul V. Biron and Ashok Malhotra. XML Schema part 2: Datatypes second edition, October 2004. W3C Recommendation. http://www. w3.org/TR/xmlschema-2/.

[3] Henning Böttger, Anders Møller, and Michael I. Schwartzbach. Contracts for cooperation between Web service programmers and HTML designers. Journal of Web Engineering, 5(1), 2006.

[4] Aske Simon Christensen, Anders Møller, and Michael I. Schwartzbach. Static analysis for dynamic XML. Technical Report RS-02-24, BRICS, May 2002. Presented at Programming Language Technologies for XML, PLAN-X'02.

[5] Aske Simon Christensen, Anders Møller, and Michael I. Schwartzbach. Extending Java for high-level Web service construction. ACM Transactions on Programming Languages and Systems, 25(6):814-875, 2003.

[6] Aske Simon Christensen, Anders Møller, and Michael I. Schwartzbach. Precise analysis of string expressions. In Proc. 10th International Static Analysis Symposium, SAS '03, volume 2694 of $L N C S$, pages 1-18. SpringerVerlag, June 2003.

[7] James Clark and Makoto Murata. RELAX NG specification, December 2001. OASIS. http://www.oasis-open.org/committees/relax-ng/.

[8] Vladimir Gapeyev, Michael Y. Levin, Benjamin C. Pierce, and Alan Schmitt. The Xtatic experience. Technical Report MS-CIS-04-24, University of Pennsylvania, October 2004. Presented at Programming Language Technologies for XML, PLAN-X '05.

[9] Matthew Harren, Mukund Raghavachari, Oded Shmueli, Michael G. Burke, Rajesh Bordawekar, Igor Pechtchanski, and Vivek Sarkar. XJ: Facilitating XML processing in Java. In Proc. 14th International Conference on World Wide Web, WWW'05, pages 278-287. ACM, May 2005.

[10] Haruo Hosoya and Benjamin C. Pierce. XDuce: A statically typed XML processing language. ACM Transactions on Internet Technology, 3(2):117$148,2003$.

[11] John B. Kam and Jeffrey D. Ullman. Monotone data flow analysis frameworks. Acta Informatica, 7:305-317, 1977. Springer-Verlag.

[12] Kohsuke Kawaguchi. Sun RELAX NG Converter, April 2003. http://www.sun.com/software/xml/developers/relaxngconverter/. 
[13] Christian Kirkegaard, Aske Simon Christensen, and Anders Møller. A runtime system for XML transformations in Java. In Proc. Second International XML Database Symposium, XSym '04, volume 3186 of LNCS. Springer-Verlag, August 2004.

[14] Christian Kirkegaard, Anders Møller, and Michael I. Schwartzbach. Static analysis of XML transformations in Java. IEEE Transactions on Software Engineering, 30(3):181-192, March 2004.

[15] Mehryar Mohri and Mark-Jan Nederhof. Robustness in Language and Speech Technology, chapter 9: Regular Approximation of Context-Free Grammars through Transformation. Kluwer Academic Publishers, 2001.

[16] Anders Møller. Document Structure Description 2.0, December 2002. BRICS, Department of Computer Science, University of Aarhus, Notes Series NS-02-7. Available from http://www.brics.dk/DSD/.

[17] Anders Møller and Michael I. Schwartzbach. The design space of type checkers for XML transformation languages. In Proc. Tenth International Conference on Database Theory, ICDT '05, volume 3363 of LNCS, pages 17-36. Springer-Verlag, January 2005.

[18] Makoto Murata, Dongwon Lee, and Murali Mani. Taxonomy of XML schema languages using formal language theory. In Proc. Extreme Markup Languages, August 2001.

[19] Henry S. Thompson, David Beech, Murray Maloney, and Noah Mendelsohn. XML Schema part 1: Structures second edition, October 2004. W3C Recommendation. http://www. w3.org/TR/xmlschema-1/. 


\section{Recent BRICS Report Series Publications}

RS-05-31 Christian Kirkegaard and Anders Møller. Type Checking with $X M L$ Schema in XACT. September 2005. 20 pp.

RS-05-30 Karl Krukow. An Operational Semantics for Trust Policies. September 2005.

RS-05-29 Olivier Danvy and Henning Korsholm Rohde. On Obtaining the Boyer-Moore String-Matching Algorithm by Partial Evaluation. September 2005. ii+9 pp. To appear in Information Processing Letters. This version supersedes BRICS RS-05-14.

RS-05-28 Jiří Srba. On Counting the Number of Consistent Genotype Assignments for Pedigrees. September 2005. 15 pp. To appear in FSTTCS '05.

RS-05-27 Pascal Zimmer. A Calculus for Context-Awareness. August 2005. 21 pp.

RS-05-26 Henning Korsholm Rohde. Measuring the Propagation of Information in Partial Evaluation. August 2005. 39 pp.

RS-05-25 Dariusz Biernacki and Olivier Danvy. A Simple Proof of a Folklore Theorem about Delimited Control. August 2005. ii+11 pp. To appear in Journal of Functional Programming. This version supersedes BRICS RS-05-10.

RS-05-24 Małgorzata Biernacka, Dariusz Biernacki, and Olivier Danvy. An Operational Foundation for Delimited Continuations in the CPS Hierarchy. August 2005. iv +43 pp. To appear in the journal Logical Methods in Computer Science. This version supersedes BRICS RS-05-11.

RS-05-23 Karl Krukow, Mogens Nielsen, and Vladimiro Sassone. $A$ Framework for Concrete Reputation-Systems. July 2005. 48 pp. This is an extended version of a paper to be presented at ACM CCS'05.

RS-05-22 Małgorzata Biernacka and Olivier Danvy. A Syntactic Correspondence between Context-Sensitive Calculi and Abstract Machines. July 2005. iv+39 pp.

RS-05-21 Philipp Gerhardy and Ulrich Kohlenbach. General Logical Metatheorems for Functional Analysis. July 2005. 65 pp.

RS-05-20 Ivan B. Damgård, Serge Fehr, Louis Salvail, and Christian Schaffner. Cryptography in the Bounded Quantum Storage Model. July 2005. 23 pp. 